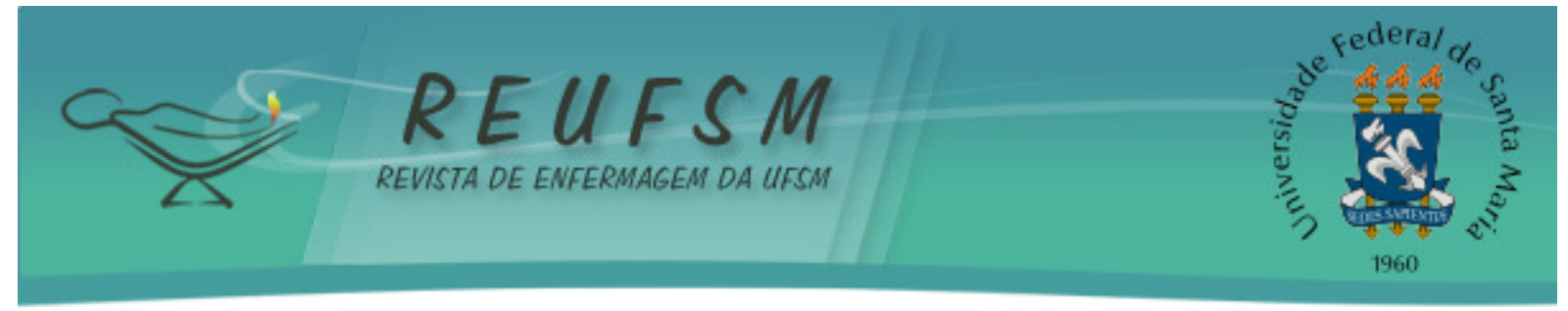

ARTIGO ORIGINAL

\title{
COMPREENSÃO DO PROCESSO DE ENFERMAGEM POR ENFERMEIROS DE UM HOSPITAL GERAL DO SUL DO BRASIL
}

\section{UNDERSTANDING OF THE NURSING PROCESS BY NURSES IN A GENERAL HOSPITAL IN SOUTHERN BRAZIL}

\section{COMPRENSIÓN DEL PROCESO DE ENFERMERÍA PARA ENFERMERAS DE UN HOSPITAL GENERAL DEL SUR DE BRASIL}

Liliane Ribeiro Trindade ${ }^{1}$ Andressa da Silveira ${ }^{2}$ Anali Martegani Ferreira ${ }^{3}$ Graciane Lafuente Ferreira ${ }^{4}$

RESUMO: Objetivo: conhecer a visão dos enfermeiros atuantes em um hospital geral da fronteira oeste do Rio Grande do Sul sobre o Processo de Enfermagem na sua prática assistencial hospitalar. Método: estudo descritivo e exploratório, com abordagem qualitativa, realizado em um hospital geral. Utilizou-se a entrevista semiestruturada com 13 enfermeiros, posteriormente os dados foram transcritos e analisados mediante a Análise de Conteúdo. Resultados: emergiram quatro categorias nesse estudo: 0 significado do Processo de Enfermagem na prática do enfermeiro; Contribuições do Processo de Enfermagem para o trabalho em equipe; Respaldo legal para o trabalho e os registros de enfermagem; Valorização do trabalho da Enfermagem. Considerações Finais: os enfermeiros reconhecem que o Processo de Enfermagem é um instrumento norteador para sua prática e que este contribui para a autonomia do profissional. Ademais, os mesmos referem que, quando o Processo de Enfermagem não é aplicado, à qualidade da assistência torna-se fragilizada.

Descritores: Enfermagem; Processos de enfermagem; Assistência de enfermagem.

ABSTRACT: Aim: to know the view of nurses that work in a general hospital in the western border of Rio Grande do Sul about the Nursing Process in their hospital care practice. Method: descriptive and exploratory study with a qualitative approach, performed in a general hospital. Semi-structured interviews with 13 nurses were used, then, the data were transcribed and analyzed using content analysis. Results: four categories emerged in this study: The meaning of the nursing process in the practice of nursing; Contributions of the Nursing Process for teamwork; Legal support for the work and nursing records; Valuation of Nursing work. Final Remarks: nurses recognized that the Nursing Process is a guiding tool for its practice and that it contributes to the professional's autonomy. Furthermore, they state that, when the nursing process is not applied, care quality becomes weakened.

Descriptors: Nursing; Nursing process; Nursing care.

\footnotetext{
1 Enfermeira. Pós-graduanda em Saúde do Trabalhador pela Universidade Federal do Rio Grande do Sul. Uruguaiana, RS, Brasil. E-mail: lilianetrindade2@gmail.com

${ }^{2}$ Enfermeira. Doutoranda em Enfermagem pela Universidade Federal de Santa Maria. Professora Assistente do Curso de Graduação em Enfermagem, Universidade Federal do Pampa. Uruguaiana, RS, Brasil. E-mail: andressadasilveira@gmail.com

3 Enfermeira. Doutora em Enfermagem. Professora Adjunta do Curso de Graduação em Enfermagem, Universidade Federal do Pampa. Uruguaiana, RS, Brasil. E-mail: analimf@gmail.com

${ }^{4}$ Discente do Curso de Graduação em Enfermagem, Universidade Federal do Pampa. Uruguaiana, RS, Brasil. Email: gralafuente@gmail.com
} 


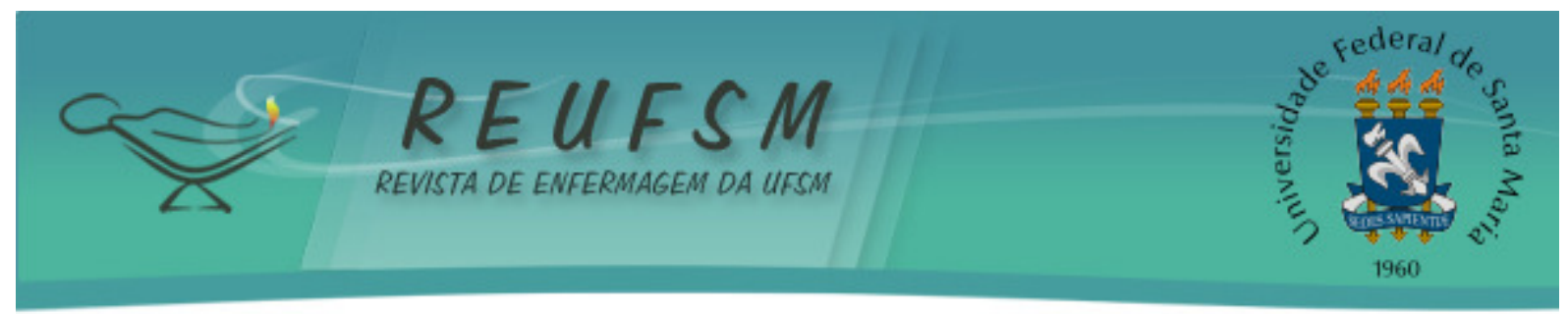

RESUMEN: Objetivo: conocer la opinión de los profesionales de enfermería que trabajan en un hospital general de la frontera occidental de Río Grande do Sul en el Proceso de Enfermería en su práctica de atención hospitalaria. Método: estudio exploratoriodescriptivo con abordaje cualitativo, realizado en un hospital general. Se utilizó entrevistas semi-estructuradas con 13 enfermeros, posteriormente los datos fueron transcritos y analizados mediante Análisis de Contenido. Resultados: cuatro categorías procedieron de este estudio: El significado del proceso de enfermería en la práctica del enfermero; Aportes del proceso de enfermería para el trabajo en equipo; Apoyo legal para los registros de trabajo y de enfermería; Valoración del trabajo de enfermería. Consideraciones finales: los profesionales de enfermería reconocen que el Proceso de Enfermería es una herramienta guía para su práctica, y que contribuye a la autonomía del profesional. Además, demuestran que, cuando el Proceso de Enfermería no es empleado, la calidad de la atención es reducida.

Descriptores: Enfermería; Proceso de enfermería; Cuidados de enfermería.

\section{INTRODUÇÃO}

A enfermagem é uma ciência que tem suas atividades centradas na manutenção e promoção da saúde, também na prevenção de doenças, por meio dos cuidados de enfermagem, com o intuito de levar às pessoas ao seu potencial máximo de saúde. ${ }^{1}$

Para o desenvolvimento da assistência de enfermagem e para subsidiar seus cuidados, os enfermeiros utilizam modelos teóricos e teorias, que objetivam relacionar fatos e estabelecer as bases da ciência de enfermagem. Os referenciais teóricos podem ser adaptados a diferentes situações clinicas e necessidades de cuidados, com pacientes, familiares e cenários de práticas. ${ }^{2}$

Para tanto, destacam-se teorias de pequeno, médio e grande alcance, as quais buscam esclarecer e subsidiar a prática de enfermagem ${ }^{3}$. Assim a estrutura do modelo teórico Padrões Funcionais de Saúde (PFS) proposta por Marjory Gordon, fornece base para avaliação clínica de enfermagem, pois apresenta 11 categorias nominais que auxiliam na identificação de diagnósticos de enfermagem, denominados de padrões funcionais de saúde. ${ }^{3}$

0 modelo padrões funcionais de saúde, constituem-se de elementos que são vistos e aplicados como conceitos centrais para a prática clínica de enfermagem e podem ser determinados pelos dados coletados na primeira etapa do Processo de Enfermagem (PE), ou seja, na coleta de dados e exame físico. Esses padrões podem ser representados a partir destes dados, sendo divididos em: percepção e controle de saúde, nutricional, metabólico, eliminações, cognitivo-perceptivo, autopercepção e autoconceito, desempenho de papel e relacionamento, sexual-reprodutivo, resposta e tolerância ao estresse, crença e valor, atividade e exercício, sono e repouso; as quais subsidiam as ações de enfermagem com vistas a atender as reais demandas de cuidado dos pacientes. ${ }^{3}$

Além da teoria padrões funcionais de saúde que permite a identificação dos padrões disfuncionais; para apoiar este estudo utilizou-se a teoria do Déficit de Autocuidado que vem subsidiar a prática do enfermeiro, para que ele seja capaz de identificar condições nas quais há um déficit na capacidade da pessoa para autocuidar-se, e para que ele possa intervir, tornando-a apta a realizar as atividades de vida de maneira satisfatória. Já os PFS discorrem que o PE possui dois elementos-chave: a identificação e a solução do problema, por meio da projeção do resultado que se espera alcançar. ${ }^{3}$

As teorias subsidiam o PE, deste modo, contribuem para a organização da Sistematização da Assistência de Enfermagem (SAE). ${ }^{1}$ A SAE organiza o trabalho quanto ao método, pessoal e instrumentos, tornando possível a operacionalização do PE como instrumento metodológico que orienta o cuidado profissional de enfermagem $e$ 


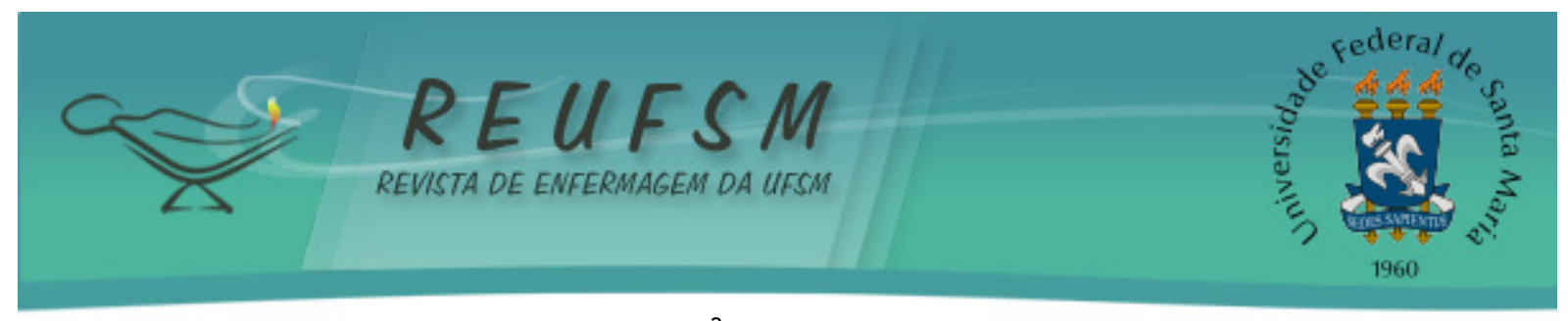

documentação da prática profissional. $^{2}$ O PE constitui-se em uma estratégia e um instrumento de trabalho do enfermeiro, desenvolvido de forma contínua para que a assistência de enfermagem seja embasada em princípios da cientificidade. ${ }^{4}$

O PE é aplicável em uma ampla variedade de situações clínicas de saúde em que as observações sobre as necessidades de cuidado dos pacientes acompanham o processo da tomada de decisão acerca do cuidado prestado e a avaliação posterior dos resultados que se obtêm com a intervenção profissional. ${ }^{5}$ Ademais, o PE tem o intuito de organizar o gerenciamento do cuidado de enfermagem, e contribuir para a tomada de decisão do enfermeiro, de forma a garantir a segurança do paciente nos diferentes níveis de atenção. ${ }^{6}$

Para isso, é imprescindível que o enfermeiro conheça os objetivos do PE, de forma que por meio de sua implementação possa atender as demandas de saúde do paciente e de sua família. Sua utilização exige conhecimentos sobre esta metodologia de trabalho, constante atualização e desenvolvimento de habilidades e experiência, orientado pela ética e padrões de conduta. ${ }^{7}$

Frente ao exposto, este estudo justifica-se pela necessidade de conhecer como os enfermeiros percebem $\mathrm{O} P \mathrm{PE}$ na sua prática assistencial hospitalar, visando a implementação deste, como metodologia de trabalho. Diante dessas premissas, objetivouse conhecer a visão dos enfermeiros sobre PE a partir de seu cotidiano em um hospital geral da fronteira oeste do Rio Grande do Sul (RS). Este estudo apresenta como questão de pesquisa: Qual a visão dos enfermeiros de um hospital geral da fronteira oeste do RS sobre o processo de enfermagem?

\section{MÉTODO}

Estudo descritivo e exploratório, com abordagem qualitativa. O cenário do estudo foi um hospital geral de um município da fronteira oeste do Rio Grande do Sul (RS).

0 hospital caracteriza-se por ser referência regional, oferta atendimentos de média e alta complexidade. Possui 230 leitos, a equipe de enfermagem conta com aproximadamente 55 enfermeiros assistenciais e 274 técnicos de enfermagem. A instituição está em fase de implementação da SAE e do PE em unidades piloto, tais como: terapia intensiva adulto e pediátrica e ambulatório de enfermagem. 0 referencial teórico utilizado para subsidiar as ações em desenvolvimento e a implementação do PE são as teorias de enfermagem Déficit no Autocuidado e Padrões Funcionais de Saúde.

Para apoiar a implementação da SAE e do PE na instituição foi criado, em 2010, um Grupo de Estudos em Saúde: com foco na Sistematização da Assistência de Enfermagem (GESSAE) em uma parceria entre o Curso de Enfermagem da Universidade Federal do Pampa (UNIPAMPA). Este grupo tem como objetivo desenvolver ações de educação permanente ${ }^{8}$, visando à qualificação da assistência, com foco na implementação da SAE e do PE.

Foram convidados a participar do estudo os enfermeiros assistenciais, com mais de três meses de trabalho na instituição a fim de que já não estivessem no período de contrato de experiência e que participassem do GESSAE. Compuseram a amostra 13 enfermeiros que atenderam aos critérios propostos, foram excluídos os enfermeiros que estivessem em período de férias e/ou licença saúde no período de coleta dos dados.

Utilizou-se entrevistas individuais com roteiro semiestruturado, realizadas em sala previamente organizada para atividade, de forma a garantir o sigilo das informações obtidas, e em horário previamente agendado, conforme disponibilidade dos enfermeiros; essas tiveram um tempo aproximado de 30 minutos, sendo gravadas em áudio.

As entrevistas foram transcritas na íntegra pela pesquisadora, e os dados foram analisados por meio da análise de conteúdo de Bardin ${ }^{9}$, que consiste em três etapas: Pré- 


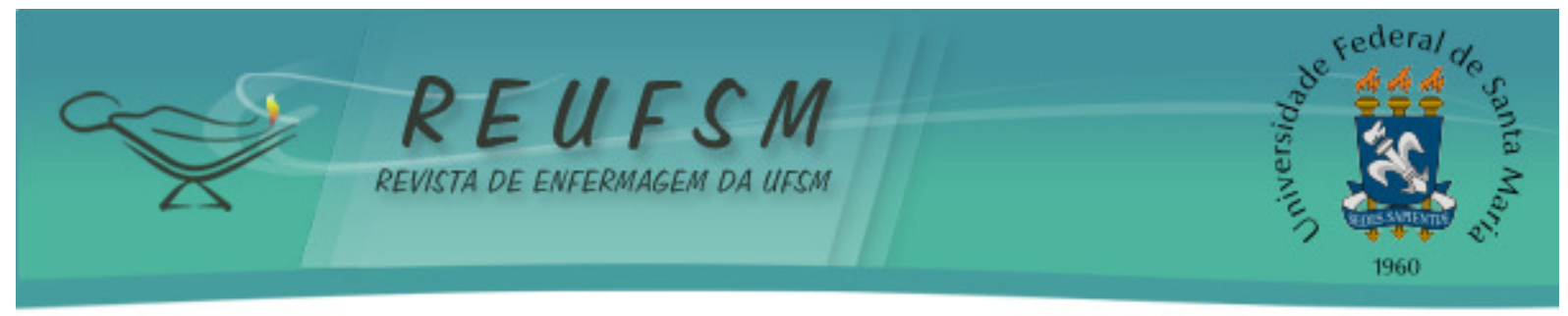

análise: consiste na seleção dos documentos, formulação das hipóteses, objetivos e identificação dos indicadores que irão nortear a interpretação final. Nesta fase ocorre a leitura flutuante, escolha dos documentos, formulação das hipóteses e objetivos, referenciação de índices, elaboração de indicadores e a preparação do material. Exploração do material: codificação das informações, permitindo uma descrição exata das características do conteúdo. A codificação compreende o recorte por temas, a enumeração de modo a utilizar a frequência para as regras de contagem e classificação e agregação dos materiais. A interpretação é realizada de modo que os conteúdos recolhidos se constituam em dados qualitativos ou análises reflexivas, em observações individuais ou gerais das entrevistas.

Para a análise foram realizadas as três etapas. Na primeira as entrevistas foram transcritas, selecionados os documentos, formulado as hipóteses e definidos os objetivos. $\mathrm{Na}$ segunda etapa foi realizada a exploração do material através de uma leitura flutuante e as entrevistas foram dividas em categorias conforme os objetivos formulados. Formaram-se quatro categorias: $O$ significado do PE na prática do enfermeiro; Contribuições do PE no trabalho em equipe; Respaldo legal para o trabalho e os registros de enfermagem; e Valorização do trabalho da enfermagem. Na terceira etapa os resultados coletados foram confrontados com a literatura atual sobre as temáticas abordadas.

0 estudo seguiu as determinações das Normas de Pesquisa em Saúde determinadas pela Resolução $n^{\circ}$ 466/12, sendo aprovado no dia 12 de Dezembro de 2013, pelo Comitê de Ética em Pesquisa (CEP) da Universidade Federal do Pampa (UNIPAMPA), sob o parecer número 490.226. Salienta-se que a coleta de dados teve início, após a aprovação do projeto pelo CEP e a assinatura do Termo de Consentimento Livre e Esclarecido em duas vias pelos participantes.

\section{RESULTADOS E DISCUSSÃO}

Participaram deste estudo 13 enfermeiros caracterizados quanto ao tempo de formação, tempo de atuação no hospital geral cenário deste estudo e sexo. 0 Quadro 1 apresenta essa distinção.

Quadro 1 - Caracterização dos sujeitos do estudo. Uruguaiana/RS. Brasil.

\begin{tabular}{|c|c|c|c|}
\hline Código & Tempo de Formação & $\begin{array}{c}\text { Tempo de trabalho } \\
\text { na Instituição }\end{array}$ & Sexo \\
\hline E1 & 5 anos & 4 anos e 10 meses & Feminino \\
E2 & 7 anos & 4 meses & Feminino \\
E3 & 8 anos & 2 anos & Feminino \\
E4 & 8 meses & 1 ano e 4 meses & Feminino \\
E5 & 2 anos e 7 meses & 1 ano e 7 meses & Feminino \\
E6 & 9 anos & 4 anos & Feminino \\
E7 & 2 anos & 1 ano & Feminino \\
E8 & 2 anos & 7 meses & Feminino \\
E9 & 2 anos e 6 meses & 2 anos e 4 meses & Masculino \\
E10 & 20 anos & 19 anos & Feminino \\
E11 & 3 anos & 3 anos & Feminino \\
E12 & 3 anos & 2 anos & Feminino \\
E13 & 4 anos & 3 anos & Feminino \\
\hline
\end{tabular}

$\mathrm{E}=$ Enfermeiro seguido de um número ordinal aleatório para representar o número de sujeitos entrevistados.

A partir das falas dos participantes desse estudo, emergiram quatro categorias que serão apresentadas em sequência. 


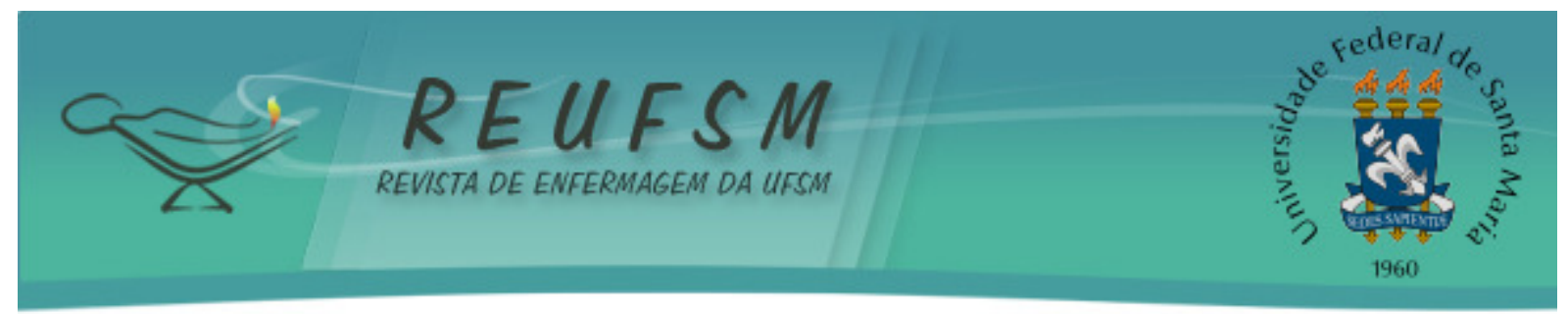

\section{O significado do Processo de Enfermagem na prática do enfermeiro}

Os enfermeiros expressaram o significado do PE na prática assistencial, como um instrumento norteador e de grande importância para a profissão. Os discursos apontam o PE como uma estratégia que proporciona respaldo legal e favorece assistência de enfermagem qualificada.

[...] É toda a estrutura do trabalho da enfermagem [...] serve para organização mesmo, para tentar organizar o serviço, o teu cotidiano. (E4)

[...] O processo vai englobar um todo, vai englobar tanto a parte técnica quanto a parte de humanização da equipe com o paciente. [...] a gente vai conseguir olhar o paciente com a visão de um todo e não uma parte específica [...] aí tu vai ver que o paciente tem outros problemas que o processo ajuda a identificar. (E10)

Serve para gente poder planejar melhor as nossas ações dentro do serviço, [...] acho que ele é válido para nosso trabalho [...] amplia os conhecimentos da gente. (E2)

[...] É o nosso instrumento de trabalho. Eu acho extremamente importante, pois a partir dele a gente vai conseguir ver o paciente desde o início da hospitalização para planejar todos os cuidados [...] (E9)

[...] então sem processo de enfermagem não existe organização $e$ não existe a própria enfermagem. (E6)

O PE possibilita alcançar resultados que são da competência e responsabilidade do enfermeiro, bem como desenvolver ações que modifiquem o estado de saúde-doença dos sujeitos. ${ }^{10}$ Indica um trabalho profissional específico e pressupõe uma série de ações dinâmicas e inter-relacionadas para sua realização, ou seja, indica a adoção de uma metodologia, fundamentada em conhecimentos técnico-científicos. ${ }^{11}$

Este leva à solução de problemas, minimiza perdas de quaisquer fatos importantes, conduz a melhores resultados com mais eficiência e menores custos, salienta a prevenção de riscos, incentivando comportamentos saudáveis, exige reflexão sobre os cuidados prestados, com foco no paciente de maneira holística e segura. ${ }^{10}$

$\mathrm{Na}$ prática assistencial, o PE impulsiona os enfermeiros a examinar suas ações, refletir como podem melhorar sua prática. ${ }^{12}$ Além disso, a utilização do PE possibilita que os enfermeiros prestem um cuidado individualizado, centrado nas necessidades de saúde do paciente; e também auxilie para a tomada de decisão. ${ }^{12} \mathrm{~A}$ avaliação permanente do $\mathrm{PE}$ promove a interação entre o enfermeiro e o paciente numa visão holística e integral. ${ }^{5}$ Sobretudo, torna-se necessário que o enfermeiro incorpore o PE como um instrumento para o desenvolvimento do seu trabalho, ou seja, cuidar com método e com a subjetividade, respeitando a individualidade do ser humano e compreendendo que o $P E$ não é um instrumento isolado do trabalho, mas sim incorporado no cuidar do enfermeiro. ${ }^{5}$ Além disso, é um instrumento de trabalho que busca ações de cuidado, identificando os 


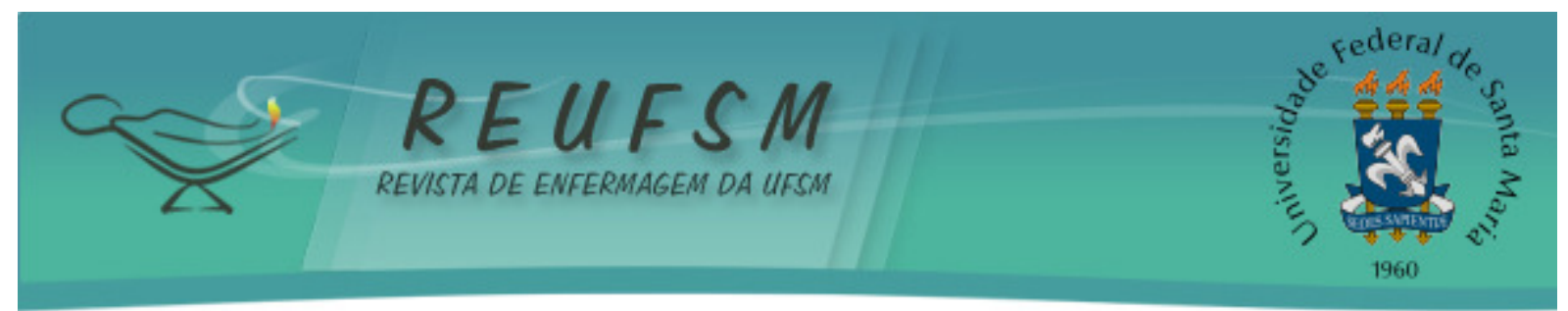

problemas de saúde, planejando e implementando suas ações e posteriormente avaliar os seus resultados. ${ }^{13}$

A teoria PFS na prática assistencial estrutura umas das etapas do PE, que vem logo após a coleta de dados. Esta etapa envolve a coleta de informações a interpretação destas informações, agrupando e formulando os diagnósticos de enfermagem. ${ }^{3}$ Mediante a temática, compreende-se que a aplicabilidade da teoria dos PFS apoia e subsidia o enfermeiro na implementação do processo de enfermagem. ${ }^{1}$

\section{Contribuições do Processo de Enfermagem para o trabalho em equipe}

A implementação e desenvolvimento do $\mathrm{PE}$, em ambiente clínico, contribui para o trabalho em equipe, visto que complementa as ações que outros profissionais realizam, focalizando as necessidades de saúde e seu impacto no cotidiano do paciente.

[...] a interligação entre médico, enfermagem e paciente no processo para ter uma boa comunicação visando esse bem-estar. (E10)

[...] fazer um bom exame físico, conversar, saber o histórico, saber o que ele [paciente] tem, isso tudo além de favorecer o nosso trabalho, [...], uma boa avaliação [...] inicial, ali já vai trazer até para o próprio médico, para o nutricionista, para o fisioterapeuta, qualquer um que chegar depois, se isso for ficar registrado pra ele saber pelo menos do que se trata esse paciente, ah, ele tem tal e tal coisa, mas ele tem histórico de hipertensão, ele já esteve internado, sei lá, diversas coisas. (E9)

[...] eu acho que dependendo da unidade onde trabalhamos, por exemplo, no terceiro andar, [que é uma unidade de internação com muitos leitos, especificidades e diferentes equipes médicas], temos que atender as demandas solicitadas pelos médicos, pelos pacientes $e$ famílias, e às vezes é desgastante [...] assim se utilizarmos o processo de enfermagem, este contribui para organizar o trabalho. (E12)

O PE promove a organização do trabalho e a comunicação efetiva entre os profissionais de enfermagem. É um meio da enfermagem comunicar-se e registrar a assistência e cuidado prestados. Auxilia na organização do trabalho em equipe, evitando a fragmentação, a qual tem sido apontada como um dos fatores que dificultam a realização do trabalho em saúde. ${ }^{14}$

A segurança do paciente é essencial ao cuidado ofertado. Nesse contexto, os enfermeiros, tem importante responsabilidade na organização do trabalho de enfermagem, visando garantir a segurança do paciente, qualidade da assistência e comunicação adequada para minimizar possíveis falhas. Para tanto, a utilização do PE enquanto metodologia, auxilia a organização das ações nos ambientes de atendimento de saúde. ${ }^{15}$

\section{Respaldo legal para o trabalho e os registros de enfermagem}

Nesta categoria são abordados os registros de enfermagem e sua importância para a prática do profissional enfermeiro.

[...] é bem importante para o bem-estar do paciente, e até pro nosso próprio respaldo. [...] Então é um respaldo legal pra ti dentro 


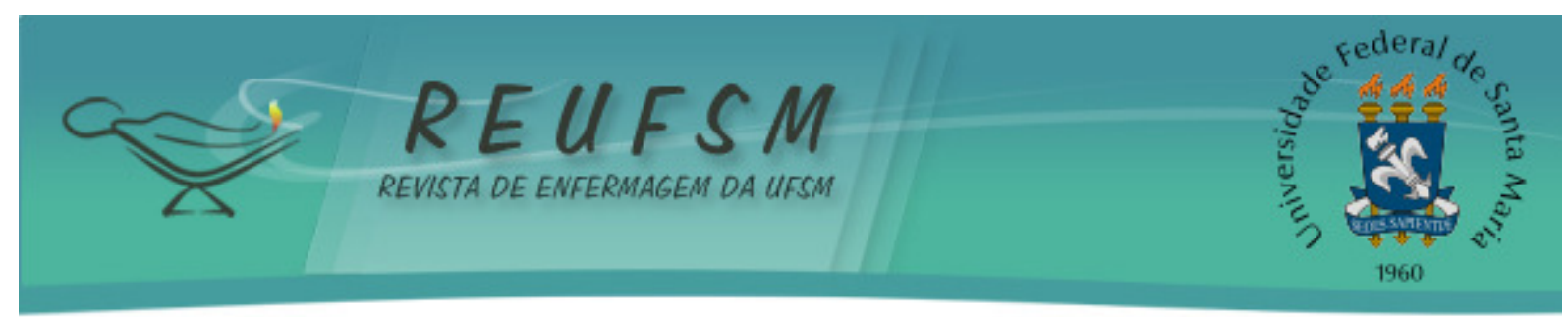

da instituição, e até pra tua vida, se tu falar nessa parte. [...] conhecendo teu paciente, tu sabe o que cada paciente tem, tu viu, tu olhou, tu fez toda essa parte, tu tem uma defesa futura de qualquer coisa [...] porque hoje em dia ainda está pouco essa parte de processo e essas coisas assim [acontecem] [...] (E9)

[...] ele é um instrumento que registra todas as ações que nós executamos na prática, o que é muito importante hoje, e, muitas vezes, nos dá até um respaldo jurídico. [...] ele também nos serve como respaldo como registro do que foi feito com esse paciente [...] (E11)

A documentação de enfermagem constitui o registro das ações na assistência ao paciente, relativo às condições do mesmo, execução dos cuidados planejados, em função de um determinado tratamento, procedimento ou diagnóstico e/ou a justificativa de não realizá-lo. São considerados documentos de enfermagem o registro de todas as fases do processo de sistematização de assistência com a finalidade clínica e administrativa. Ademais, esses registros são válidos, se estiverem registrados de forma adequada em documento apropriado. ${ }^{16}$

No processo de atenção à saúde, a documentação das atividades e seus resultados por meio de registros escritos constituem-se como instrumento de comunicação efetivo para o planejamento, continuidade e avaliação da assistência, além de ser fonte de informações para questões jurídicas, de pesquisa e educação. ${ }^{17}$

Apesar da importância dos registros de enfermagem em relação aos aspectos legais e assistenciais, estes com frequência não contêm informações adequadas para apoiar a instituição, a equipe e o paciente em questões judiciais. ${ }^{17} \mathrm{~A}$ falta de registros de enfermagem ou a realização do registro de forma inadequada demonstram uma assistência descomprometida com o cuidado, além de trazer danos para a instituição de saúde. ${ }^{18}$

Os registros de enfermagem fazem parte do amparo legal da profissão. São caracterizados como anotações de enfermagem, destinadas a toda equipe de saúde e são fundamentais para a efetivação do PE ou sistematização da assistência. ${ }^{19}$

Esses registros são fontes de documentação das ações e atividades exercidas pela equipe de enfermagem, tornando-se uma forma de garantir e comprovar a prestação do cuidado e a qualidade da assistência prestada pela equipe de enfermagem. Eles ainda fornecem informações específicas para a continuidade do cuidado, além de subsidiar a elaboração de um plano assistencial efetivo e individualizado. Registrar as ações realizadas pela equipe indica a qualidade da assistência que está sendo prestada e comprova a boa prática profissional, além do cumprimento ético e legal. ${ }^{17}$

\section{Valorização do trabalho da Enfermagem}

No contexto atual dos serviços de saúde, há uma grande procura dos usuários por serviços que ofereçam assistência segura e de qualidade, e que estão atentos aos eventos adversos que podem ocorrer durante a assistência à saúde. Logo, os profissionais da saúde também procuram ambientes de trabalho que ofereçam satisfação e segurança ao desempenho de suas funções. ${ }^{20}$

A motivação para o trabalho ocorre por meio da satisfação que o ambiente oferta e pela realização profissional, que são necessidades inerentes dos trabalhadores enfermeiros, mas que estão relacionadas direta ou indiretamente com o respeito e o reconhecimento profissional. ${ }^{21}$ 


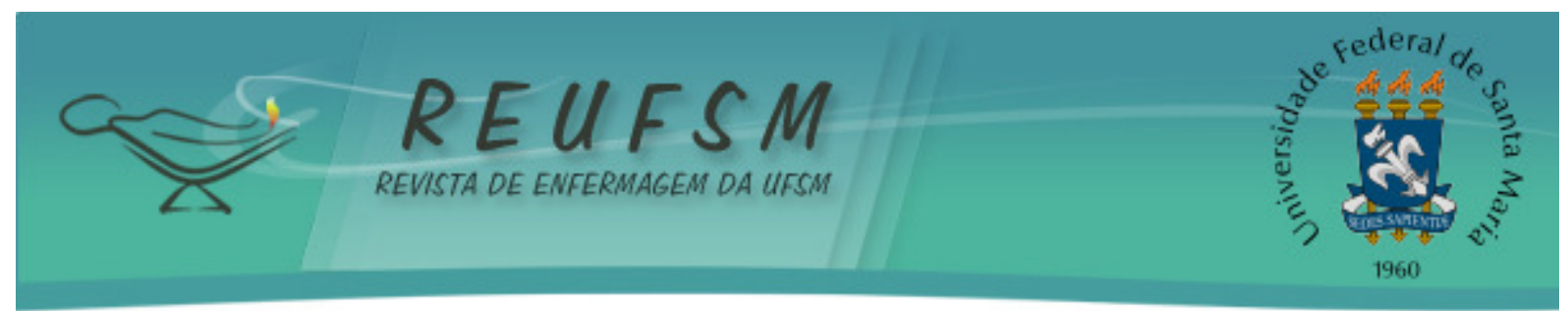

Todavia a maioria dos serviços de saúde não disponibiliza recursos humanos e financeiros suficientes para atender às necessidades dos trabalhadores e usuários dos serviços. Isso acarreta sobrecarga de trabalho aos profissionais inseridos nos serviços de saúde. ${ }^{20}$

Com certeza, o processo é importante, mas apesar de que na nossa prática a gente muitas vezes não consegue dar conta, né, [...] porque muitas vezes, por exemplo [...] eu tô em mais de uma unidade [...] (E1)

Dentre os profissionais de saúde, os de enfermagem estão mais sujeitos a essa sobrecarga e desvalorização do trabalho. Os enfermeiros expressaram a necessidade de valorização da profissão na instituição.

[...] além de valorizar o serviço de enfermagem, né, acho que é muito importante para a valorização do nosso serviço. (E4)

Eu acho muito importante, eu acho que a gente tem que enfatizar cada vez mais os profissionais [de enfermagem], tem que ter mais conhecimento sobre esses processos, para poder desenvolver melhores trabalhos [...], melhores trabalhos que eu digo em função do paciente, na organização da unidade, pois dessa forma a unidade e o profissional vão ser mais valorizados [...]. (E6)

Nesse sentido, a Política Nacional de Humanização (PNH) propõe que os serviços de saúde garantam uma gestão participativa aos seus trabalhadores, de forma a promover ações de incentivo e valorização da jornada integral de trabalho, bem como a participação em processos de educação permanente que possam qualificar a inserção dos trabalhadores no Sistema Único de Saúde (SUS). ${ }^{22}$ Contudo, em muitas organizações de saúde há desvalorização dos trabalhadores de saúde e pouca participação na gestão dos serviços. ${ }^{21}$

Isso implica no desgaste, do processo de trabalho dos profissionais da saúde, o que pode repercutir também na insatisfação dos profissionais de enfermagem para o exercício de suas funções, levando à desmotivação no trabalho. ${ }^{23}$ Esse fator pode provocar a desestruturação na organização do trabalho da enfermagem. ${ }^{24}$

Apesar das fragilidades das organizações de saúde, as quais contribuem para a desvalorização do trabalho dos enfermeiros, cabe à enfermagem organizar ações que viabilizem a valorização e legitimação profissional no trabalho. ${ }^{2}$

Diante disso, a valorização e o reconhecimento do trabalho desempenhado pelo enfermeiro é de suma importância para sua satisfação em um ambiente de trabalho seguro, de respeito mútuo, com oportunidades para o desempenho de suas funções. A necessidade de se valorizar o trabalhador, sua participação no processo decisório, o incentivo do potencial criativo, a satisfação de suas necessidades, a humanização das relações de trabalho e a melhoria das condições laborais, só trarão benefícios para o paciente, pois isso se refletirá na forma do cuidado ao paciente. ${ }^{25}$

\section{CONSIDERAÇÕES FINAIS}

Por meio deste estudo foi possível compreender o significado do PE para os enfermeiros, e como percebem sua implementação e aplicabilidade na prática assistencial.

Os enfermeiros reconhecem o PE como um instrumento norteador que proporciona qualidade na prática assistencial e logo, contribui na valorização e na autonomia dos 


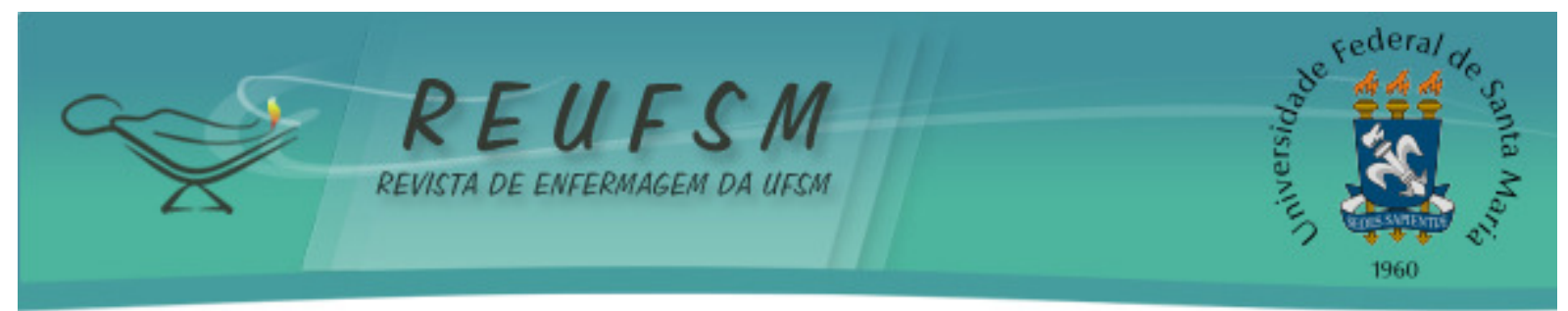

profissionais. Pode-se ainda, destacar que quando o PE não é realizado adequadamente, a qualidade da assistência pode ficar comprometida.

Considera-se que o PE é um caminho para a autonomia da profissão, por constituir-se em uma metodologia de trabalho reconhecida pelos enfermeiros, que permite aproximação do profissional com o paciente, equipe e familiares, e possibilita também a comunicação entre os diferentes profissionais da equipe de saúde. Também exige conhecimento científico e compromisso ético com o exercício da profissão.

A baixa adesão à realização do PE entre os enfermeiros entrevistados ocorreu em sua maioria, devido a repetição de um trabalho fragmentado, tempo reduzido para o desenvolvimento das atividades de sua competência, ou ainda, pelo desconhecimento dos benefícios da utilização do PE.

Entretanto, destaca-se que o estímulo institucional e da gerência de enfermagem para capacitar os profissionais para o uso desta tecnologia são fatores que podem facilitar para adesão a este método de trabalho, e assim auxiliar no trabalho do enfermeiro. A utilização do PE na instituição contribuirá na qualidade do cuidado, e para a visibilidade e reconhecimento do enfermeiro.

Ressalta-se que o PE contribui significativamente para a qualidade da assistência e do cuidado de enfermagem. Contudo, faz-se necessário fomentar pesquisas na área, sendo relevante a interação entre academia e as instituições de saúde, consolidando parcerias e firmando espaços para o desenvolvimento de atividades de ensino, pesquisa e extensão que contemplem o PE.

Cabe salientar a importância da realização de novos estudos com esta temática, para confirmar estes achados e qualificar a assistência de enfermagem, uma vez que este estudo representa um recorte de uma região onde não há implementação do PE.

\section{REFERÊNCIAS}

1. Horta WA. Processo de enfermagem. Rio de Janeiro: Guanabara Koogan; 2011.

2. Conselho Federal de Enfermagem. Resolução COFEN n 358, de 15 de outubro de 2009. Processo de enfermagem em ambientes, públicos ou privados. Dispõe sobre a Sistematização da Assistência de Enfermagem e a implementação do Processo de Enfermagem em ambientes, públicos ou privados, em que ocorre o cuidado profissional de Enfermagem, e dá outras providências \{Internet]. [acesso em 2014 jan 29]. Disponível em: http: / / www.diariodasleis.com.br/busca/exibelink.php?numlink=212736.

3. Pereira CDFD, Tourinho FSV, Ribeiro JLS, Medeiros SBM, Santos VEP. Padrões funcionais de saúde: diagnósticos de enfermagem em escolas da rede pública. Texto \& Contexto Enferm [Internet]. 2013 [acesso em 2014 jan 15];22(4):1056-63. Disponível em: http://www.scielo.br/scielo.php?pid=S0104-07072013000400023\&script=sci_arttext.

4. Gordon M. Nursing diagnosis: process and application. $3^{\text {rd }}$ ed. St. Louis: Mosby; 1994.

5. Souza MFG, Santos ADB, Monteiro Al. O processo de enfermagem na concepção de profissionais de Enfermagem de um hospital de ensino. Rev Bras Enferm [Internet]. 2013 [acesso 2014 jan 15];66(2):67-173. Disponível em: http://www.scielo.br/scielo.php?script=sci_arttext\&pid=S0034-71672013000200003.

6.Soares MI, Terra FS, Oliveira LS, Resck ZMR, Esteves AMSD, Moura CC. Processo de enfermagem e sua aplicação em unidade de terapia intensiva: revisão integrativa. Rev Enferm UFPE [Internet]. 2013 [acesso em 2014 jan 15];7 Esp:4183-91. Disponível em: http://www.revista.ufpe.br/revistaenfermagem/index.php/revista/article/viewFile/3242/ pdf_2617. 


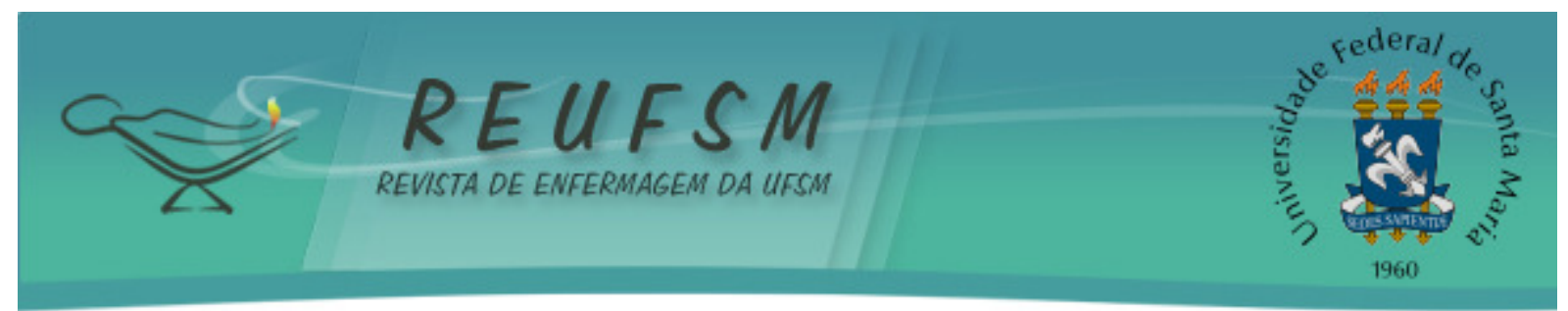

7. Silva EGC, Oliveira VC, Neves GBC, Guimarães TMR. O conhecimento do enfermeiro sobre a Sistematização da Assistência de Enfermagem: da teoria à prática. Rev Esc Enferm [Internet]. 2011 [acesso em 2014 jan 21];45(6):1380-6. Disponível em: http://www.scielo.br/pdf/reeusp/v45n6/v45n6a15.pdf.

8. Sarreta, FO. Educação permanente em saúde para os trabalhadores do SUS [online]. São Paulo: Editora UNESP, Cultura Acadêmica; 2009 [acesso em 2013 maio 18]. ISBN 978-857983-009-9. Disponível em: http://books.scielo.org/id/29k48. p. 248.

9. Bardin L. Análise de conteúdo. Lisboa: Edições 70; 2009.

10. Alfaro-Lefevre R. Aplicação do processo de enfermagem: uma ferramenta para o pensamento crítico. $7^{\mathrm{a}}$ ed. Porto Alegre: Artes Médicas; 2010.

11. Garcia TR, Nóbrega MML. Processo de enfermagem: da teoria à prática assistencial e de pesquisa. Esc Anna Nery [Internet]. 2009 [acesso em 2014 jan 22];13(1):188-93. Disponível em: http://www.eean.ufrj.br/revista_enf/20091/ARTIG0\%2024.pdf.

12. Trigueiro EV, Leite JEL, Dantas DNA, Coura AS, Enders BC. Perfil e posicionamento do enfermeiro gerente quanto ao processo de enfermagem. Esc Anna Nery [Internet]. 2014 [acesso em 2014 jan 22];18(2):343-9. Disponível em: http://www.scielo.br/scielo.php?pid=S1414-81452014000200343\&script=sci_arttext.

13. Pereira MJB, Fortuna CM, Mishima SM, Almeida MCP, Matumoto S. A enfermagem no Brasil no contexto da força de trabalho em saúde: perfil e legislação. Rev Bras Enferm [internet]. 2009 [acesso em 2014 jan 22];62(5):771-7. Disponível em: http: / / www.scielo.br/scielo.php?pid=S0034-71672009000500022\&script=sci_arttext.

14. Matos E, Pires DEP, Campos GWS. Relações de trabalho em equipes interdisciplinares: contribuições para a constituição de novas formas de organização do trabalho em saúde. Rev Bras Enferm [Internet]. 2009 [acesso em 2014 jan 29];62(6):863-9. Disponível em: http: //www.scielo.br/pdf/reben/v62n6/a10v62n6.pdf.

15. Lima SBS, Rabenschlag LA, Tonini TFF, Menezes FL, Lampert AN. Conflitos gerenciais e estratégias de resolução pelos enfermeiros gerentes. Rev Enferm UFSM [Internet]. 2014 [acesso em 2014 jan 26];4(2):419-28. Disponível em: http://cascavel.ufsm.br/revistas/ojs2.2.2/index.php/reufsm/article/view/11888/pdf.

16. Conselho Regional de enfermagem. Decisão COREN/RS n 115, de 30 de agosto de 2006. Normatiza no Estado do Rio Grande do Sul os princípios gerais para ações que constituem a documentação de enfermagem [Internet]. [acesso em 2014 jan 1]. Disponível em: http://www.portalcoren-

rs.gov.br/docs/Legislacoes/legislacao_d9497d5565bff743e2fe204ba85580c7.pdf.

17. Françolin L, Brito MFP, Gabriel CS, Monteiro TM, Bernardes A. A qualidade dos registros de enfermagem em prontuários de pacientes hospitalizados. Rev Enferm UERJ [Internet]. 2012 [acesso em 2014 jan 15];20(1):79-83. Disponível em: http://www.facenf.uerj.br/v20n1/v20n1a14.pdf.

18. Setz VG, Innocenzo MD. Avaliação da qualidade dos registros de enfermagem no prontuário por meio da auditoria. Acta Paul Enferm [Internet]. 2009 [acesso em 2014 jan 24];22(3):313-7. Disponível em: http://www.scielo.br/pdf/ape/v22n3/a12v22n3.pdf.

19. Claudino HG, Gouveia EML, Santos SR, Lopes MEL. Auditoria em registros de enfermagem: revisão integrativa de literatura. Rev Enferm [Internet]. 2013 [acesso em 


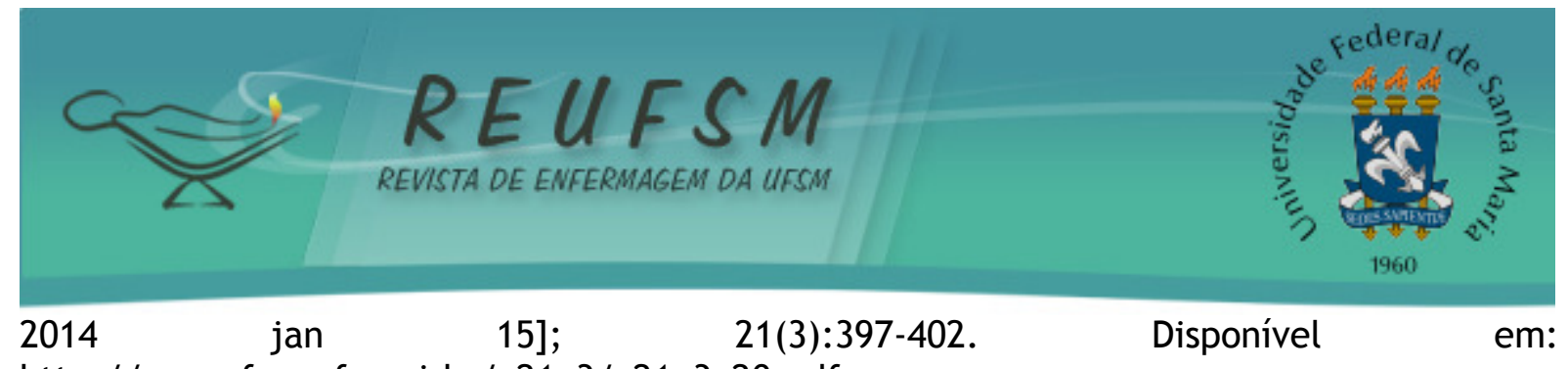

http://www.facenf.uerj.br/v21n3/v21n3a20.pdf.

20. Mendes IAC, Trevizan MA, Mazzo A, Godoy S, Ventura CAA. Marketing profissional e visibilidade social na enfermagem: uma estratégia de valorização de recursos Humanos. Texto \& Contexto Enferm [Internet]. 2011 [acesso em 2014 jan 24];20(4):788-95. Disponível em: http://www.scielo.br/pdf/tce/v20n4/19.pdf.

21. Sprandel LIS, Vaghetti HH. Valorização e motivação de enfermeiros na perspectiva da humanização do trabalho nos hospitais. Rev Eletrônica Enferm [Internet]. 2012 [acesso em 2014 jan 24];14(4):794-802. Disponível em: http://www.revistas.ufg.br/index.php/fen/article/view/16100/13343.

22. Brasil. Ministério da Saúde. Portaria $n^{\circ} 198$ GM/MS, de 13 de fevereiro de 2004. Institui a Política Nacional de Educação Permanente como estratégia do Sistema Único de Saúde para a formação e o desenvolvimento de trabalhadores para o setor e dá outras providências. Brasília (DF); 2004 [acesso em 2014 jan 24]. Disponível em: http://dtr2001.saude.gov.br/sas/PORTARIAS/Port2004/GM/GM-198.htm.

23. Meneghini F, Paz AA, Lautert L. Fatores ocupacionais associados aos componentes da síndrome de Burnout em trabalhadores de enfermagem. Texto \& Contexto Enferm [Internet]. 2011 [acesso em 2014 jan 24];20(2):225-33. Disponível em: http://www.scielo.br/pdf/tce/v20n2/a02v20n2.

24. Vaghetti HH. As perspectivas de um retrato da cultura organizacional de hospitais públicos brasileiros: uma tradução, uma bricolagem [tese]. Florianópolis (SC): Universidade Federal de Santa Catarina; 2008. 223 p.

25. Daubermann DC, Tonete VLP. Qualidade de vida no trabalho do enfermeiro da Atenção Básica à Saúde. Acta Paul Enferm [Internet]. 2012 [acesso em 2014 jan 24];25(2):277-83. Disponível em:

http:// www.scielo.br/scielo.php?pid=S010321002012000200019\&script=sci_arttext.

Data de recebimento: $16 / 10 / 2014$

Data de aceite: 23/04/2015

Contato do autor responsável: Liliane Ribeiro Trindade

Endereço postal: Doutor Maia- 2475- Bairro: Centro- CEP: 97501-724 Uruguaiana/RS

E-mail: lilianetrindade2@gmail.com 